\title{
Large Deformation Modeling of Wing-Like Structures Based on Support Vector Regression
}

\author{
Chao An ${ }^{1} \oplus$, Changchuan Xie ${ }^{1, *}$, Yang Meng ${ }^{1}$, Xiaofei Shi ${ }^{2}$ and Chao Yang ${ }^{1}$ \\ 1 School of Aeronautics Science and Engineering, Beihang University, Beijing 100191, China; \\ Ac@buaa.edu.cn (C.A.); summy@buaa.edu.cn (Y.M.); yangchao@buaa.edu.cn (C.Y.) \\ 2 Department of UAV General Technology, HiWing General Aviation Equipment co.LTD, \\ Beijing 100074, China; xiaofshi@foxmail.com \\ * Correspondence: xiechangc@buaa.edu.cn
}

Received: 9 July 2020; Accepted: 26 August 2020; Published: 29 August 2020

\begin{abstract}
Large flexible aircrafts produce large deformation during flight, leading to obvious geometric nonlinearities. Large deformation modeling is essential for modern aircraft design. Calculation of large deformation based on a full-order model often carries an unbearable computing burden. The reduced-order model (ROM) can be efficient in calculation but requires lots of test datasets. This study investigates support vector regression (SVR) to build a regression model to calculate the static large deformation of wing-like structures. The correlation coefficient $(\mathrm{R})$ and root mean square error (RMSE) are used to evaluate the performance of the regression models. In contrast to the ROM that has been proposed, the regression model based on SVR requires far fewer training cases, with almost the same accuracy in this research. Meanwhile, comparison with another prediction model built based on random forest regression (RFR) has also been reported. The results reveal that the SVR algorithm has better accuracy on this issue. Finally, ground test results of a real large flexible wing model show that the regression model proposed here reaches a good agreement with measurement data under applied load. This work illustrates that the machine learning algorithm is an efficient and accurate way to predict large deformation of aircrafts.
\end{abstract}

Keywords: large deformation; geometric nonlinearity; support vector regression; reduced-order models

\section{Introduction}

With the development of modern, high-performance aircrafts, structure flexibility is getting larger and larger to meet the requirements of high flight performance. Geometric nonlinearities must be considered in the aircrafts' design. Large deformation subjected to the aerodynamic forces during flight leads to essential effects on stiffness characteristics and aerodynamic configuration, which obviously affect stability, dynamic response and flight performance [1-5]. Accurate calculation on large deformation is of great significance in modern aircrafts' design [6]. Full-order models, like the finite element method (FEM) in physical degrees of freedom (DoFs), often carry a high computational burden, especially for complex wing-like structures $[7,8]$. With geometric nonlinearities, the linear modal techniques cannot be used directly to dramatically simplify the structural model. High computational burden gradually becomes the key factor restricting large deformation simulation, especially in aeroelastic or optimization analysis.

A reduced-order model (ROM) based on a modal form with test cases is an attractive theory for large deformation economic calculation. Several authors have applied the approach in static or dynamic response simulation in the recent past [9-17]. A review of ROM methods for geometric nonlinear structures was presented by Mignolet et al. [18]. ROM methods considering geometric 
nonlinearities require a nonlinear stiffness expression given in advance. Mignolet [9] used quadratic and cubic terms of the basis function to describe nonlinear stiffness. For determination of stiffness coefficients in ROM, two approaches can be chosen. The first one, which is called the direct method, is intrusive, as it explicitly expresses the nonlinear stiffness coefficients [18]. But, the stiffness coefficients are not available in most commercial FEM source. For the complex model, the approach should be impractical [19]. The second one, which is called the indirect method, is non-intrusive, and is the most used method in ROM construction. In this approach, a series of load cases is specified, and the corresponding deformations are calculated by a nonlinear FEM software package, like MSC.Nastran or Abaqus. The nonlinear stiffness coefficients can be solved by a least squares regression analysis through test cases, including load cases and deformations. The accuracy of ROM depends on the choice of test cases $[13,20]$. Load vector in test cases must ensure that the desired nonlinear effects are excited in the whole procedure $[15,21]$. Due to the requirement and characteristics proposed, the construction of ROM needs lots of test cases to ensure the calculation accuracy. Even the calculation procedure of ROM is much faster than the full-order model, but a lot of time will be spent in obtaining test cases.

Support vector regression (SVR) is a powerful nonlinear regression approach, which belongs to the support vector machine (SVM) $[22,23]$. SVR introduces a nonlinear kernel to model the nonlinear function of multiple variables and uses insensitive loss function to accept small deviations from objective function. Compared to other regression techniques, SVR is based on the structural risk minimization principle [24] so that it matches the available training sample reasonably well, and generalizes well to the new testing dataset $[25,26]$. In this regard, SVR has better generalization capacity and is also suitable for small sample size regression. Chang et al. pointed out that SVR performs relatively better with less samples and more features [27]. With the least square regression approach used in ROM described before, empirical risk minimization is employed to force the regression model to converge to the sample target value as much as possible, without consideration of the structural characteristics of the regression model [25]. Traditional ROM needs lots of datasets to meet the accuracy requirements. SVR has been proven effective for model regression in engineering applications [28-33]. However, rare research is proposed before applying the SVR method into large deformation prediction of flexible structures.

Motivated by prior studies, the SVR method is applied into large deformation calculation of flexible wing-like structures in this paper. We first obtained a series of test cases by the commercial FEM software package as training samples. Second, we used SVR to construct prediction models of static large deformation. The accuracy of prediction models was validated by comparing to nonlinear FEM calculation results and real structure test results. Meanwhile, another machine learning algorithm, random forest regression (RFR) [34], was also investigated in prediction model construction, providing a comparison with the SVR algorithm. Finally, ROM was constructed as the traditional large deformation simulation technique with the same samples. The contrast of number of test cases required for SVR and ROM with the same accuracy was presented. The correlation coefficient $(\mathrm{R})$ and root mean square error (RMSE) were applied properly to evaluate the performance of the prediction models.

The rest of this paper is organized as follows. Details of the theory and method are presented in Section 2. Before the SVR method, the background of the physical problem and the traditional ROM approach are also briefly reviewed. The performance contrast of the traditional ROM and SVR methods proposed is presented in this paper. Section 3 shows the structure model and data used in the simulation. Ground deformation test measuring techniques are also introduced. Section 4 gives the validation results and the performance evaluations of the prediction models. Conclusions are shown in Section 5. 


\section{Theory and Method}

\subsection{Structrue Dynamics}

The equation of motion of the structure, including geometric nonlinearities, is often given in the dynamic equation as [14]:

$$
\frac{\partial\left(F_{i j} S_{j k}\right)}{\partial X_{k}}+\rho_{0} b_{i}^{0}=\rho_{0} \ddot{u}_{i}(i, j, k=1,2,3)
$$

where tensor $S$ is the second Piola-Kirchhoff stress tensor and tensor $F$ is the deformation gradient tensor, $\rho_{0}$ is the mass density of the structure, $b^{0}$ is the vector of the body force and subscripts $i, j, k$ distinguish the elements in corresponding tensors. $X$ denotes the position vector of the structure. With discretizing by the FEM, the structural dynamic equation can be described as:

$$
M \ddot{u}(t)+K u(t)+f_{N L}(u(t))=f(t)
$$

where $M$ and $K$ are the linear mass and stiffness matrices in physical space, $u(t)$ is the deformation vector, $f_{N L}$ represents the nonlinear restoring force and $f(t)$ is the external force. The structural damp is ignored in this research.

The linear vibration modes of the structure can be obtained by solving the degenerate form of Equation (2):

$$
\left(K-\omega_{i}^{2} M\right) \varphi_{i}=0
$$

where $\omega_{i}$ are the mode frequencies and $\varphi_{i}$ denote the corresponding mode vectors.

Giving the assumption:

$$
u(t)=\Gamma q(t)
$$

where $\Gamma=\left(\varphi_{1}, \varphi_{2}, \cdots, \varphi_{m}\right)$ is the mode shape matrix, $\varphi_{i}, i=1,2, \cdots, m$ are mass normalized mode shape vectors and $q(t)=\left(q_{1}, q_{2}, \cdots, q_{m}\right)^{T}$ is the generalized coordinates vector in modal space. The mode shape matrix $\Gamma$ is truncated to a small set of modal shape. The DoFs of the structural dynamic equation can be reduced to a small number, $m$. Substituting Equation (4) into Equation (2), the dynamic equation is given as:

$$
\ddot{q}(t)+\Lambda q(t)+S\left(q_{1}, q_{2}, \cdots, q_{m}\right)=\widetilde{f}(t)
$$

where $\Lambda$ is the $m \times m$ diagonal stiffness matrix in modal space, $\widetilde{f}(t)=\left(\widetilde{f_{1}}(t), \widetilde{f_{2}}(t), \cdots, \widetilde{f_{m}}(t)\right)^{T}$ is the modal excitation force and $S=\left(S^{1}, S^{2}, \cdots S^{m}\right)^{T}$ is the nonlinear function of all generalized coordinates. Each equation in the reduced dynamic equation is formulated as:

$$
\ddot{q}_{i}(t)+\omega_{i} q_{i}(t)+S^{i}\left(q_{1}, q_{2}, \cdots, q_{m}\right)=\widetilde{f_{i}}(t), \quad i=1,2, \cdots, m
$$

For the static problem, the acceleration term $\ddot{q}(t)$ is ignored:

$$
\begin{gathered}
\Lambda q(t)+S\left(q_{1}, q_{2}, \cdots, q_{m}\right)=\widetilde{f}(t) \\
\omega_{i} q_{i}(t)+S^{i}\left(q_{1}, q_{2}, \cdots, q_{m}\right)=\widetilde{f_{i}}(t), \quad i=1,2, \cdots, m
\end{gathered}
$$

The difficulty of construction of a large deformation prediction model is to determine the expression of the nonlinear function $S$. In a traditional nonlinear structural ROM, the nonlinear function is given by [16]:

$$
S^{i}=\sum_{j=1}^{m} \sum_{k=1}^{m} a_{j k}^{i} q_{j} q_{k}+\sum_{j=1}^{m} \sum_{k=1}^{m} \sum_{t=1}^{m} b_{j k t}^{i} q_{j} q_{k} q_{t}, \quad i=1,2, \cdots, m
$$


where $a^{i}, b^{i}$ are the coefficients and the nonlinear function is assumed as the quadratic and cubic polynomial formulation of generalized coordinates. With test cases obtained by nonlinear FEM, coefficients $a^{i}, b^{i}$ can be solved by least square regression analysis [14]. When coefficients $a^{i}, b^{i}$ are solved, the generalized coordinates can be calculated with Equation (8) to recover the large deformation. For better predicting capacity of large deformation, the SVR method is used to determine the nonlinear relationship, S, of Equation (7) in this research instead of the definite nonlinear formulation and least square regression analysis in traditional ROM.

\subsection{Support Vector Regression}

For a given dataset, $G=\left\{\left(x_{1}, y_{1}\right),\left(x_{2}, y_{2}\right), \cdots,\left(x_{N T}, y_{N T}\right)\right\}$, where $x_{i}=\left(x_{i 1}, x_{i 2}, \cdots, x_{i d}\right)^{T} \in \mathbb{R}^{d}$ is the $i$ th training sample with feature dimensionality of $d, x_{i j}$ is the value of the $j$ th feature, $y_{i} \in \mathbb{R}$ is the regression target value of the sample and NT is the number of training samples. In the linear situation, SVR is used to find a decision function [35]:

$$
f(x)=\langle\omega, x\rangle+b
$$

where $\omega \in \mathbb{R}^{d}$ is a normal vector of the hyper-plane, $b$ is the bias parameter and $\langle$,$\rangle represents the dot$ product. The optimization objective is to minimize the risk function as follows:

$$
R=\frac{1}{2}\|\omega\|^{2}+c \sum_{i=1}^{N T}|\xi|_{\varepsilon}\left(f\left(x_{i}\right)-y_{i}\right)
$$

where $c$ is a regularization constant and $\varepsilon$ is the deviation from each sample target value $y_{i}$, which is also the given threshold. $|\xi|_{\varepsilon}$ is an $\varepsilon$-insensitive loss function, given as $[25,35]$ :

$$
|\xi|_{\varepsilon}=\left\{\begin{array}{l}
0, \text { if }|\xi| \leq \varepsilon \\
|\xi|-\varepsilon, \text { otherwise }
\end{array}\right.
$$

In order to describe the real deviation from the sample target value to the fitting value, two slack variables, $\bar{\xi}_{i}, \xi_{i}$, which tackle the infeasible constraints are introduced, and the minimizing problem can be solved as:

$$
\begin{array}{r}
R=\frac{1}{2}\|\omega\|^{2}+c \sum_{i=1}^{N T}\left(\xi_{i}+\bar{\xi}_{i}\right) \\
\text { s.t. }\left\{\begin{array}{l}
y_{i}-\left\langle\omega, x_{i}\right\rangle-b \leq \varepsilon+\xi_{i} \\
\left\langle\omega, x_{i}\right\rangle+b-y_{i} \leq \varepsilon+\bar{\xi}_{i} \\
\xi_{i}, \bar{\xi}_{i} \geq 0
\end{array}\right.
\end{array}
$$

The first term of Equation (13) describes the flatness of the objective function, which is called "structural risk", and the second term describes the fitness between the function and actual sample target values, which is called "empirical risk". When constant $c$ equals to zero, the minimizing problem goes back to linear regression, which also means that the rule is too strict [25]. The schematic diagram of SVR has been shown in Figure 1.

The dual problem of Equation (13) can be derived using the Lagrange function. Additionally, $\omega$ can be defined as an integration of training patterns of $x$, linearly. The decision function can be reformulated as [36]:

$$
f(x)=b+\sum_{i}^{N T}\left(\alpha_{i}-\bar{\alpha}_{i}\right)\left\langle x_{i}, x\right\rangle
$$

where $\alpha_{i}, \bar{\alpha}_{i}$ are the Lagrangian multipliers. 


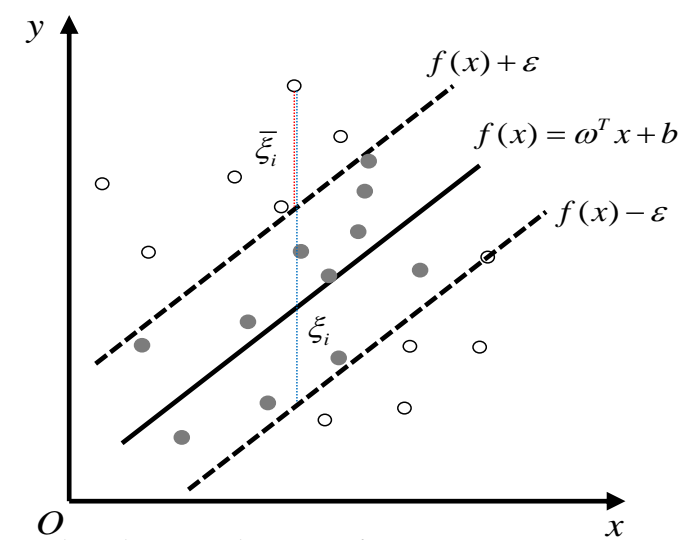

Figure 1. The schematic diagram of support vector regression (SVR).

For the nonlinear problems, it is possible to find an objective function that also means a hyper-plane to satisfy both good flatness and fitness simultaneously. We can obtain a nonlinear SVR model by replacing the dot product with a nonlinear kernel function. The kernel function can be introduced as:

$$
K\left(x, x^{\prime}\right)=\left\langle\Phi(x), \Phi\left(x^{\prime}\right)\right\rangle
$$

where $\Phi(x)$ represents a map from the sample space to the feature space. The principle of kernel mapping in the nonlinear SVR model is shown in Figure 2. With the kernel function, the decision function of nonlinear SVR can be formulated as:

$$
f(x)=b+\sum_{i}^{N T}\left(\alpha_{i}-\bar{\alpha}_{i}\right) K\left(x, x_{i}\right)
$$

The Gaussian kernel is widely used in nonlinear SVR, given by:

$$
K\left(x, x_{i}\right)=\exp \left(-\gamma\left\|x-x_{i}\right\|^{2}\right)
$$

Parameters $\gamma$ and $c$ are required to be selected in construction of the nonlinear SVR.

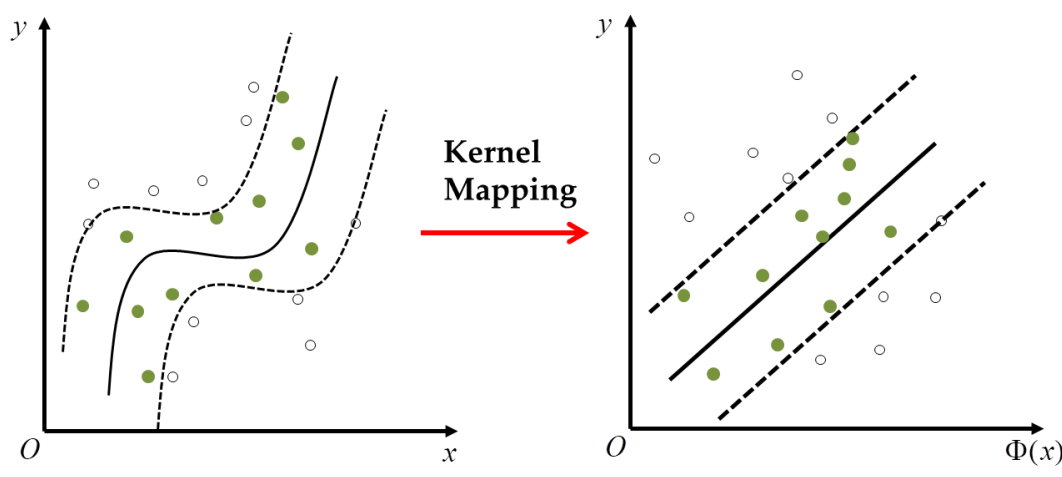

Figure 2. The principle of kernel mapping in nonlinear SVR model.

\subsection{SVR Prediction Model of Large Deformation}

Based on the SVR model, the static prediction model for large deformation of flexible wing-like structure can be established. The modal excitation force is defined as a feature and the generalized coordinates in modal space are given as the target value. The dataset is formulated as:

$$
G^{r}=\left\{\left(\widetilde{f_{1}}, q_{r, 1}\right),\left(\widetilde{f_{2}}, q_{r, 2}\right), \cdots,\left(\widetilde{f}_{N T}, q_{r, N T}\right)\right\} \quad r=1,2, \cdots m
$$


where $m$ is the order of modal space, NT is the number of samples, which are also called test cases, and $q_{r}$ is scalar and denotes the r-th generalized coordinates in modal space. For a modal space with $m$ modal shapes, the number of SVR models required is also $m$.

Choosing of samples is important for the prediction model. The samples include given loads and corresponding deformations, which is the same as traditional ROM construction. Deformations are generated in nonlinear FEM software. Two factors are important in choosing the given load in samples. The first is the spatial distribution of the load over the FEM model. The second is the overall magnitude of the FEM loading. With the absence of a priori information about the exact nature of the nonlinear relationship, a sum of a number of weighted modal shapes is often used as load in samples:

$$
\begin{gathered}
\tilde{f}=\Gamma\left(\sum_{r=1}^{m} a_{r} \lambda_{r} \varphi_{r}\right) \\
\lambda_{r}=\frac{2 i}{g-1}-1, \quad i=0,1, \cdots, g-1
\end{gathered}
$$

where $a_{r}$ are scalar weighting factors for each selected modal shape and $g$ is grid density applied to define intermediate loads in order to have a wide range of sampling space.

The Gaussian kernel is used as a kernel function in this research to build the nonlinear SVR model. After samples are obtained, the suitable parameters regularization $c$ in Equation (13) and kernel parameters $\gamma$ are selected by classical grid search techniques [30]. A simple grid search, with grids value from $2^{-5}$ to $2^{15}$ for $c$ and $2^{-13}$ to $2^{3}$ for $\gamma$, both in interval $2^{1}$, is used for parameters selection. $\varepsilon$ is given empirically before $(c, \gamma)$ selection and those parameters are assumed fixed in the SVR model.

The samples are divided into training samples and test datasets. The training sample is used for establishment of the SVR model and the test dataset is used for assessing the performance of the SVR model. The procedure illustration of the SVR model's construction is presented in Figure 3.

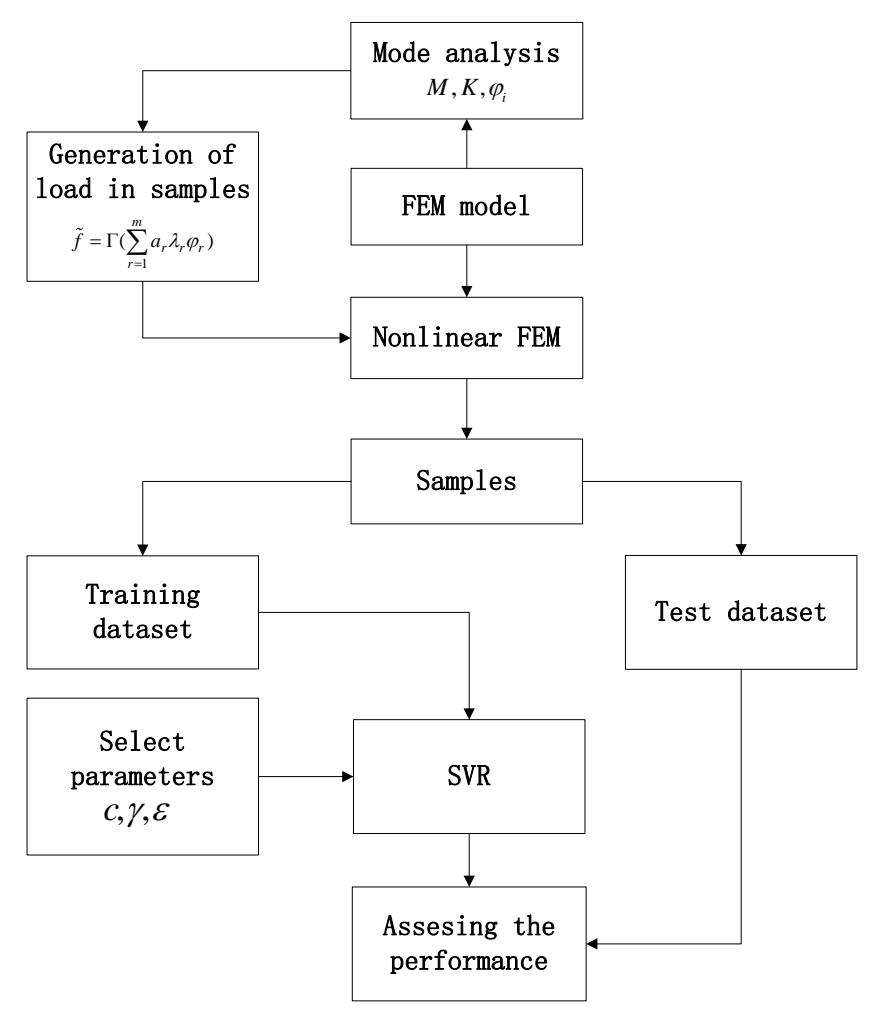

Figure 3. Procedure of SVR model construction. 
Once the prediction model has been built and tested, static deformation in modal space can be solved after giving any force input. The deformation in physical space of wing-like structures can be obtained by Equation (4).

\section{Structure Model and Data}

A large flexible wing structure model is presented here to demonstrate the accuracy and efficiency of the prediction model proposed for static deformation calculation. A real wing model has also been completed to validate the accuracy of the SVR model in practical application through a ground deformation test.

\subsection{Wing Model}

The parameters of the large flexible wing model are presented in Table 1. The CATIA model, FEM model (MSC.Nastran) and real model in the ground test are shown in Figures 4 and 5. In this wing model, a rectangular steel ruler was chosen as the beam and the rectangular section is $35 \times 1.5 \mathrm{~mm}$. Each section attaches to the wing beam at a single point. A sufficient amount of clearance is left between each section to ensure that no stiffness will be added to the wing beam by the external shell.

Table 1. Design parameters of the wing model.

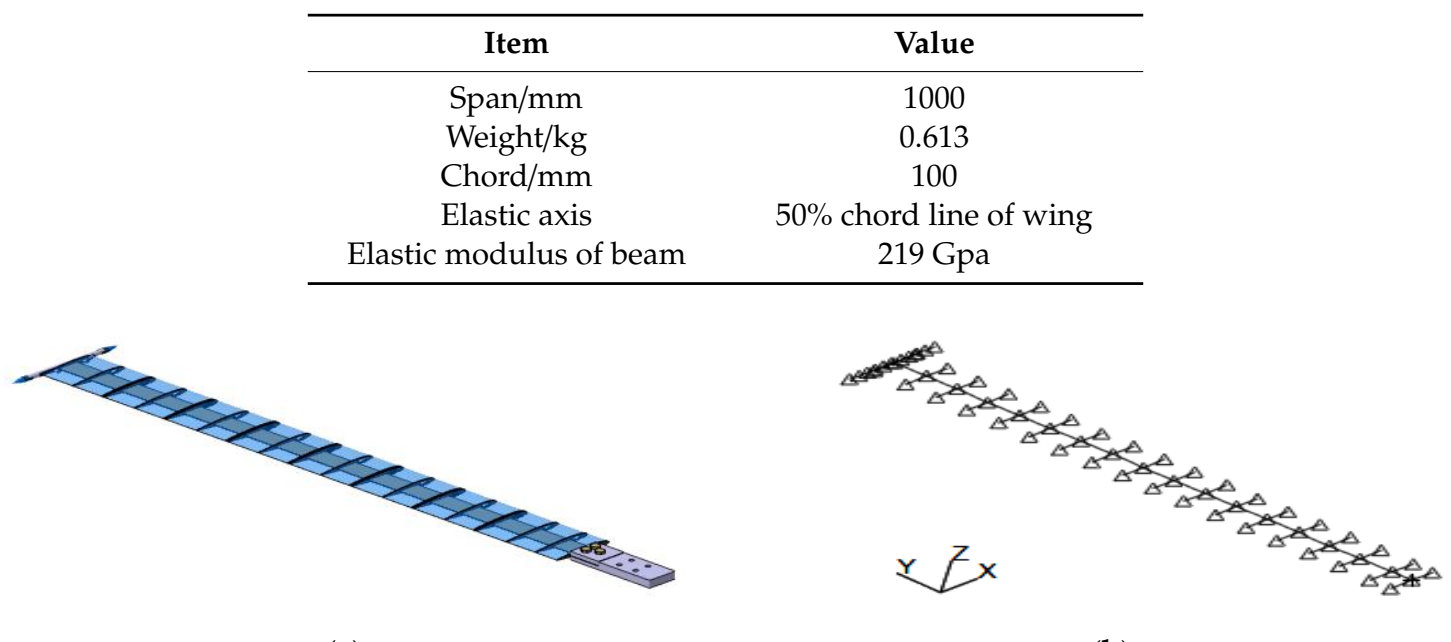

(a)

(b)

Figure 4. Model of wing. (a) Three-dimensional (3D) CATIA model (b) finite element method (FEM) model.

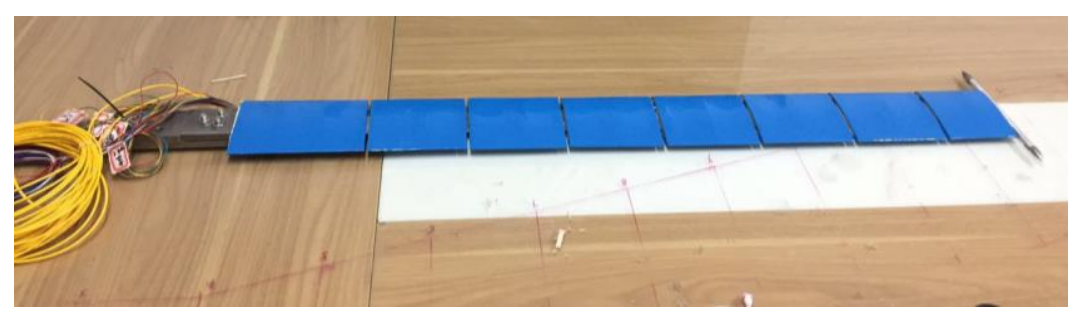

Figure 5. Model in ground deformation test.

Table 2 lists the first five linear modes of the wing model with a fixed root condition. The results obtained from the FEM analysis show good agreement with ground vibration test (GVT) results. The linear mode frequency of the first vertical bending is quite low, indicating high flexibility of the wing. 
Table 2. First five linear modes and frequencies (in $\mathrm{Hz}$ ) of the wing model. GVT = ground vibration test.

\begin{tabular}{cccc}
\hline Number & Mode & FEM Analysis Results & GVT Results \\
\hline 1 & First vertical bending & 1.179 & 1.18 \\
2 & Second vertical bending & 7.724 & 7.81 \\
3 & Third vertical bending & 22.19 & 22.35 \\
4 & First torsion & 22.95 & 23.07 \\
5 & First horizontal bending & 27.47 & 27.63 \\
\hline
\end{tabular}

\subsection{Sample Dataset for SVR}

The sample datasets used in this research were created in MSC.Nastran after determining load by Equation (20). After applying load on the wing model structure, corresponding deformation in physical space can be calculated directly. Generalized coordinates, which are also the modal coordinates, can be obtained by Equation (4). In this research, the modal space includes 4 modes and the dataset includes 270 samples. Four modal loads, $\widetilde{f_{1}}, \widetilde{f_{2}}, \widetilde{f_{3}}, \widetilde{f_{4}}$, are taken as features and four generalized coordinates, $q_{1}, q_{2}, q_{3}, q_{4}$, as decision variables. The distribution of vertical deformation of the wing tip is shown in Figure 6 by sample numbers.

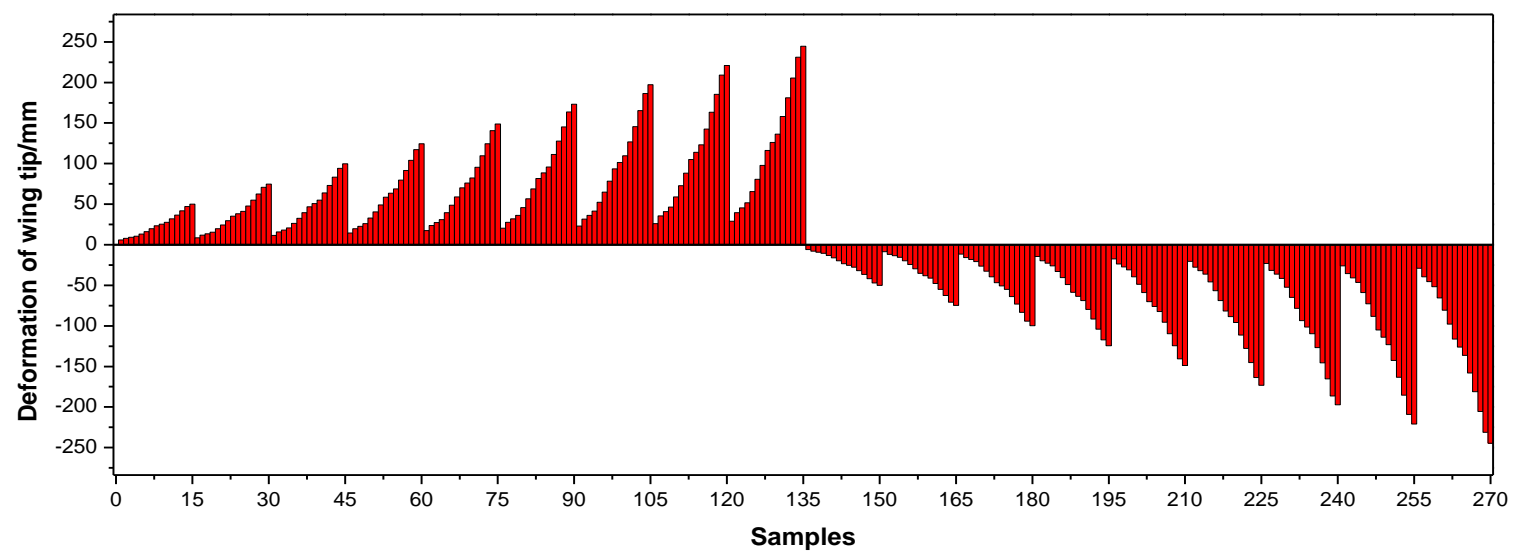

Figure 6. Samples of deformation.

The maximum of wingtip deformation in samples is about $250 \mathrm{~mm}$, which is $25 \%$ of the wing-span length. It satisfies the requirement of nonlinear analysis. The distributions of the first four generalized coordinates are shown in Figures 7-10 by sample numbers. They have the same tendency as the distribution of deformation in physical space. The sign of the value depends on the modal shape form.

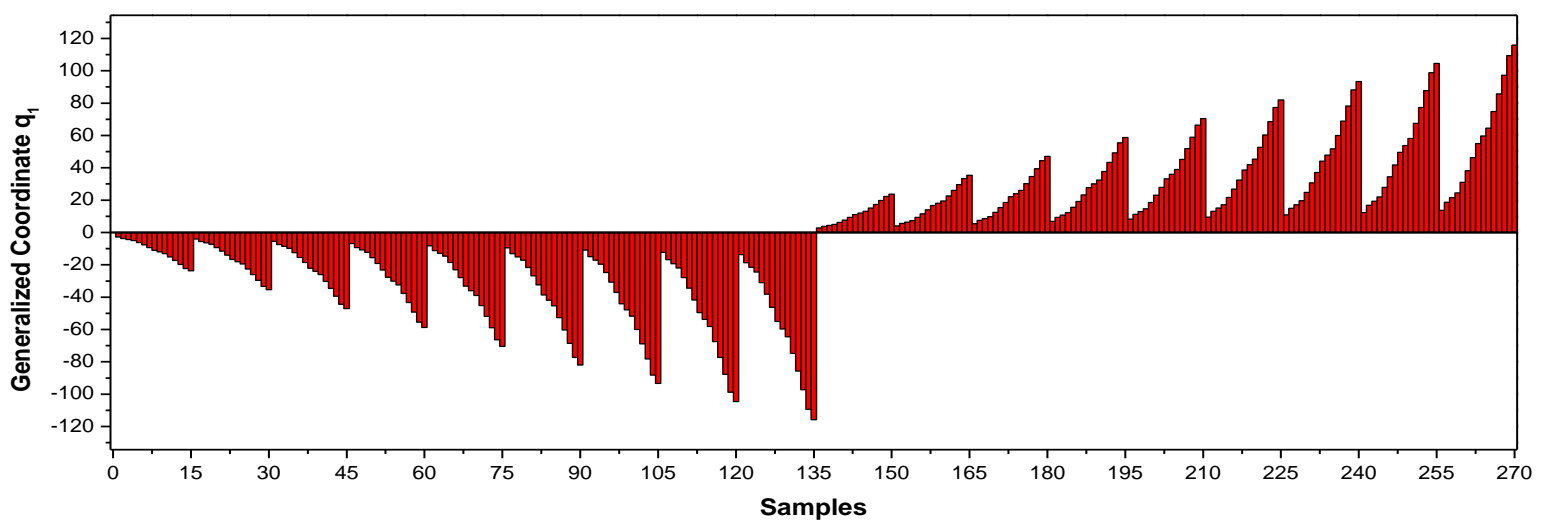

Figure 7. Samples of first-order generalized coordinate. 


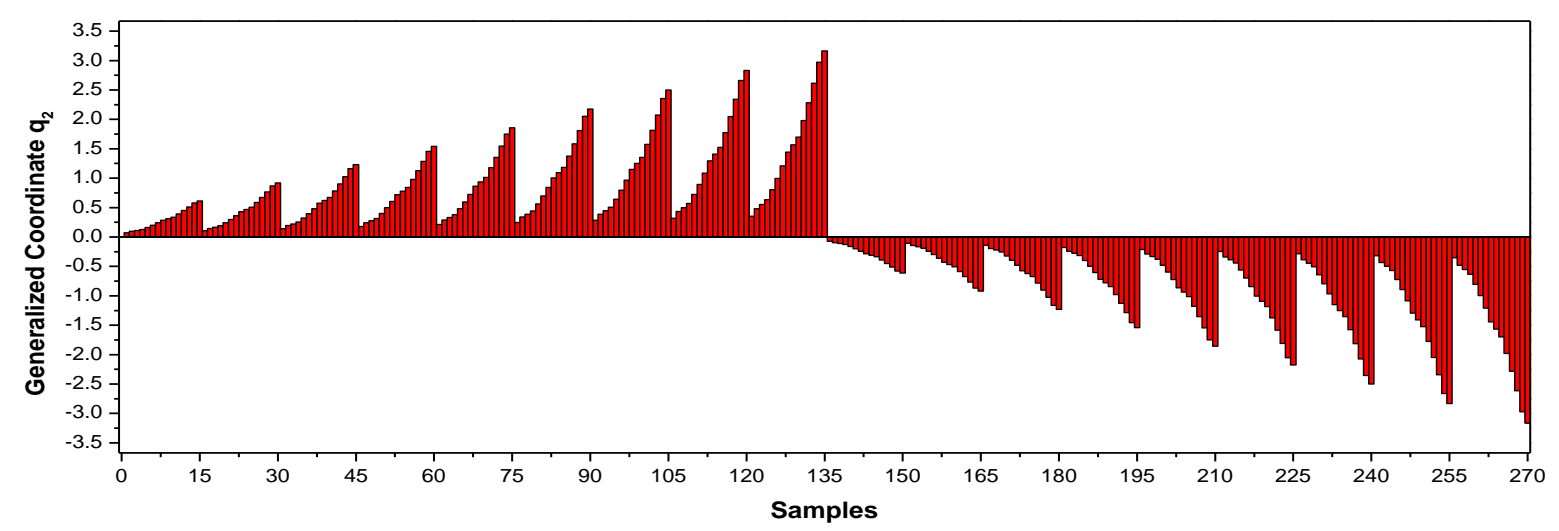

Figure 8. Samples of second-order generalized coordinate.

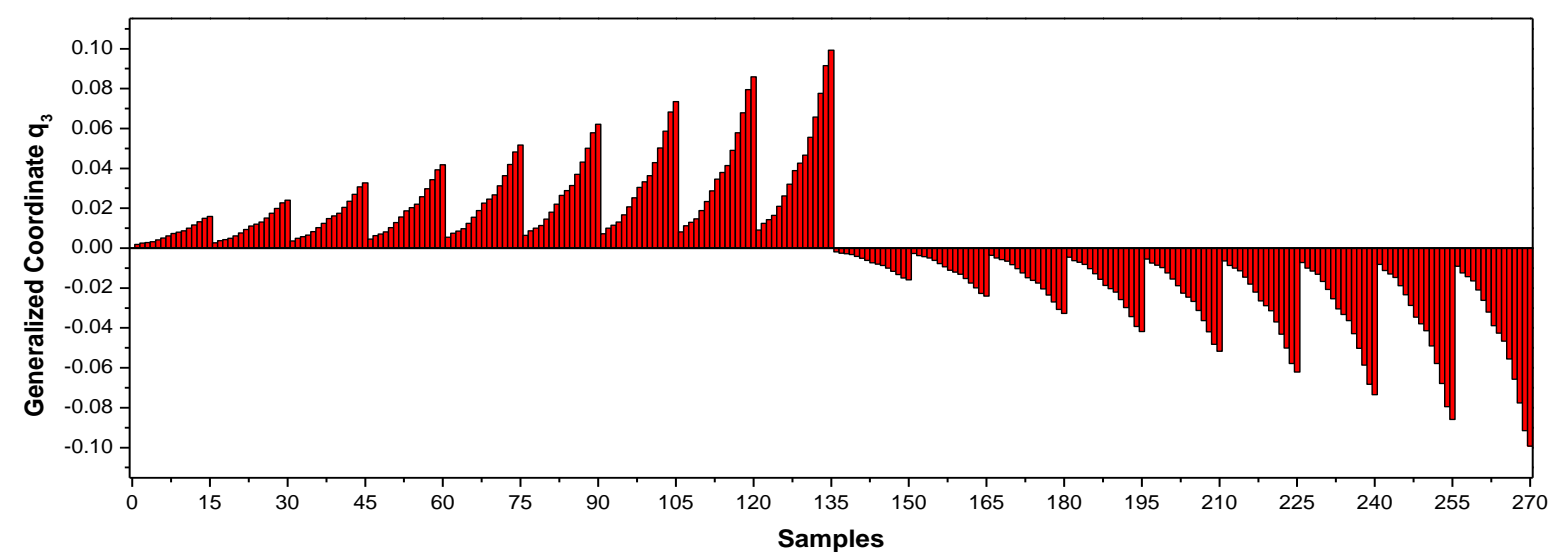

Figure 9. Samples of third-order generalized coordinate.

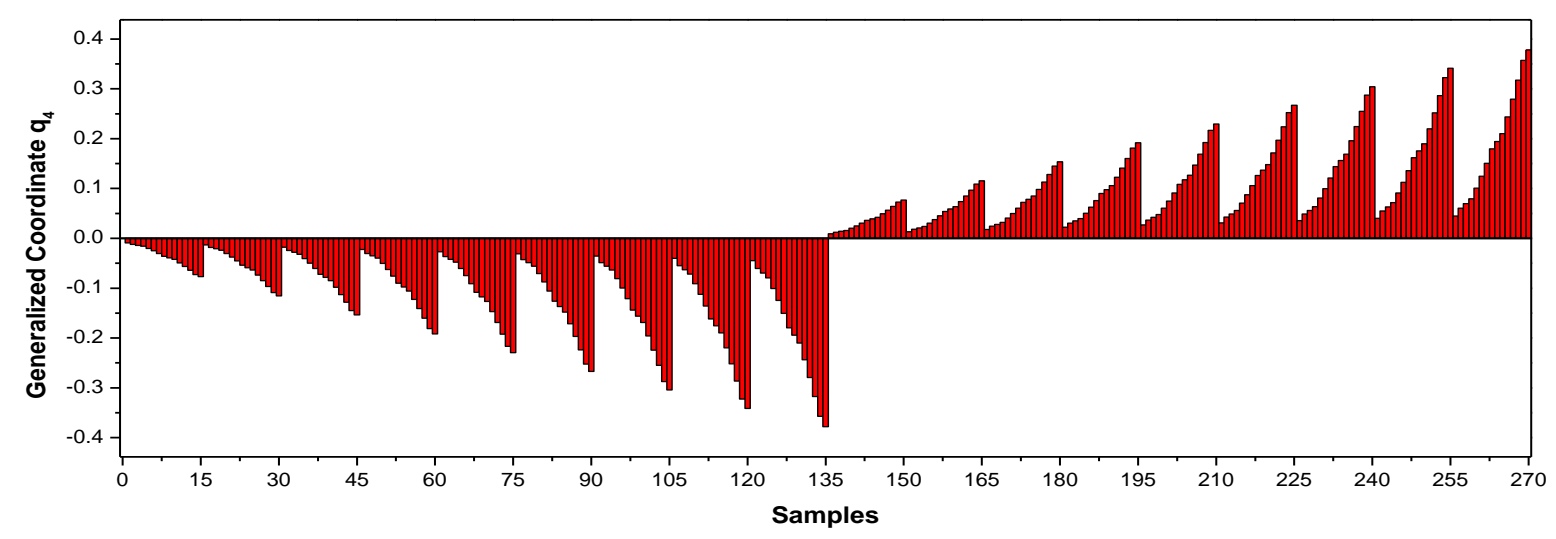

Figure 10. Samples of fourth-order generalized coordinate.

\subsection{Ground Deformation Test Measuring Techniques}

Measuring structural large deformation accurately and efficiently is a usually big challenge. In the research proposed here, an advanced fiber optic sensing system is introduced to measure the structural large deformation in an indirect way [37]. Fiber grating (FBG) sensors with different characteristic wavelengths are attached to the wing, as shown in Figures 11 and 12. The FBG sensors can measure strains and are arranged at different locations so that strain values can be obtained at these stations. After strain values are measured, the deformation can be calculated by numerical integration with a strain-displacement transformation (SDT) [37,38]. 


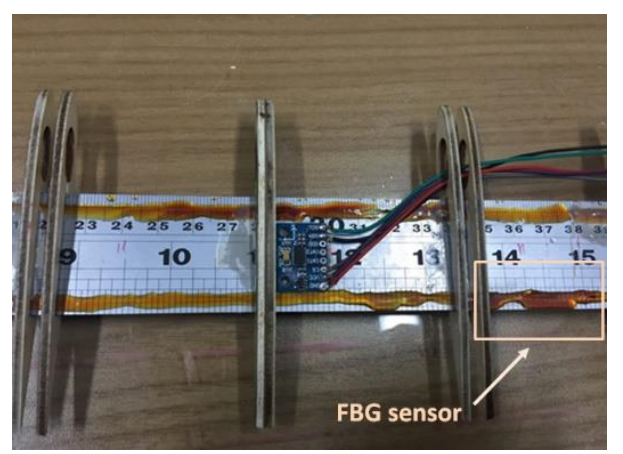

Figure 11. Fiber grating (FBG) sensor on the wing model.

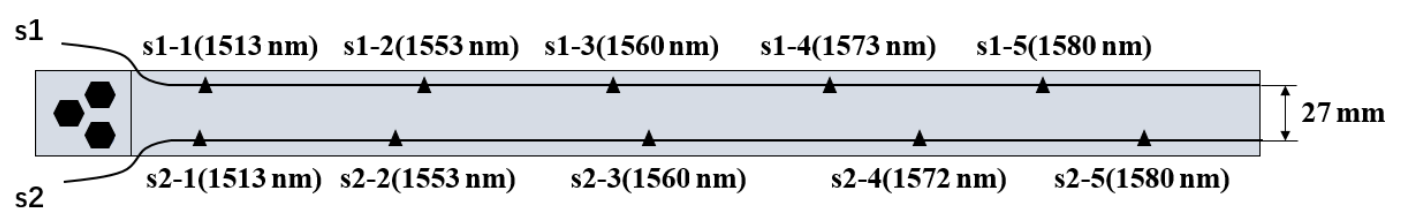

Figure 12. Arrangement of FBG sensors (wavelength in bracket).

\section{Results and Discussion}

The SVR model was established to predict the static large deformation of the flexible wing model. In this research, 70\% (190 samples) of the sample dataset described in Section 3.2 was used to train the proposed method as a training sample, and the remaining 30\% (80 samples) was used for testing the performance. After training and testing, correlation coefficient (R) and root mean squared error (RMSE) were used to evaluate the method as statistical performance indices. Each of the indices are calculated as:

$$
\begin{gathered}
\mathrm{R}=\frac{\sum_{i=1}^{N T}\left(x_{i}-\bar{x}\right)\left(y_{i}-\bar{y}\right)}{\sqrt{\sum_{i=1}^{N T}\left(x_{i}-\bar{x}\right)^{2} \sum_{i=1}^{N T}\left(y_{i}-\bar{y}\right)^{2}}} \\
\text { RMSE }=\sqrt{\frac{1}{N T} \sum_{i=1}^{N T}\left(x_{i}-y_{i}\right)^{2}}
\end{gathered}
$$

where $x_{i}, y_{i}$ represent the actual sample value and predicted value. $\bar{x}, \bar{y}$ represent the mean of actual values and predicted values. Based on the correlation coefficient, R, SVR parameter $c$ is chosen as 32 , $\gamma$ is chosen as $1 / 16$ and $\varepsilon$ is given as 0.05 . Figures $13-16$ present the prediction results with the test dataset. The order of test data samples has been rearranged based on the prediction value, from smallest to largest.

The prediction results show the excellent correlation coefficient between actual values with a black solid line and predicted values with a red circle symbol. With this SVR model, the generalized coordinates can be predicted accurately for excited load input. Translating the coordinates from modal space to physical space by Equation (4), the actual and predicted values in test datasets of wingtip vertical deformation are shown in Figure 17. Predicted values reached good agreement with actual values. The SVR model proposed in this research has good performance in large deformation calculation of the flexible wing model. 


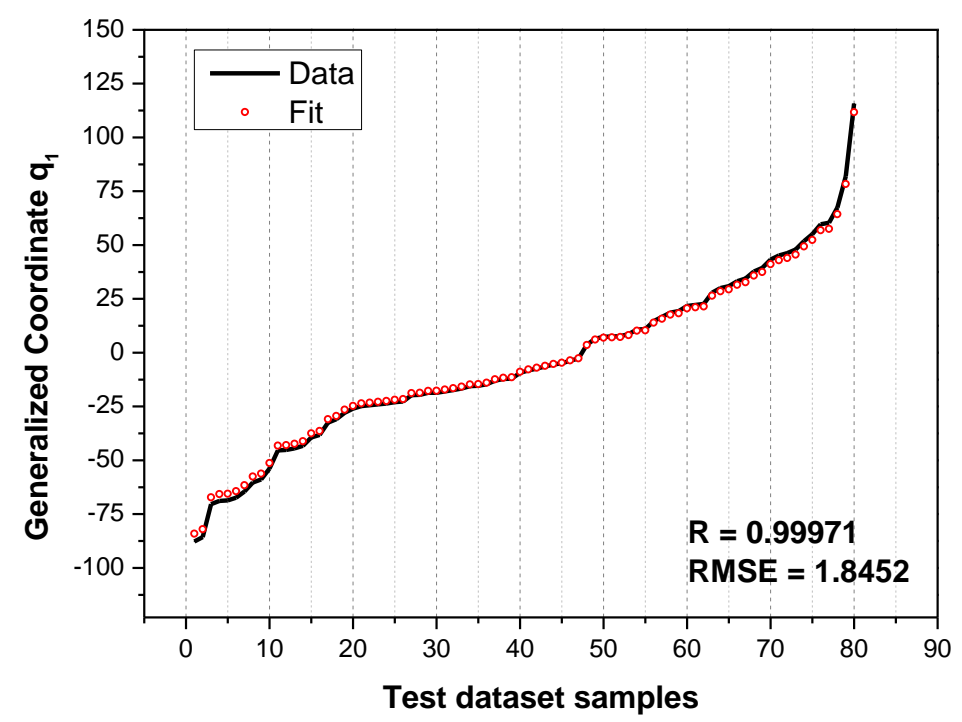

Figure 13. Prediction results of first generalized coordinate.

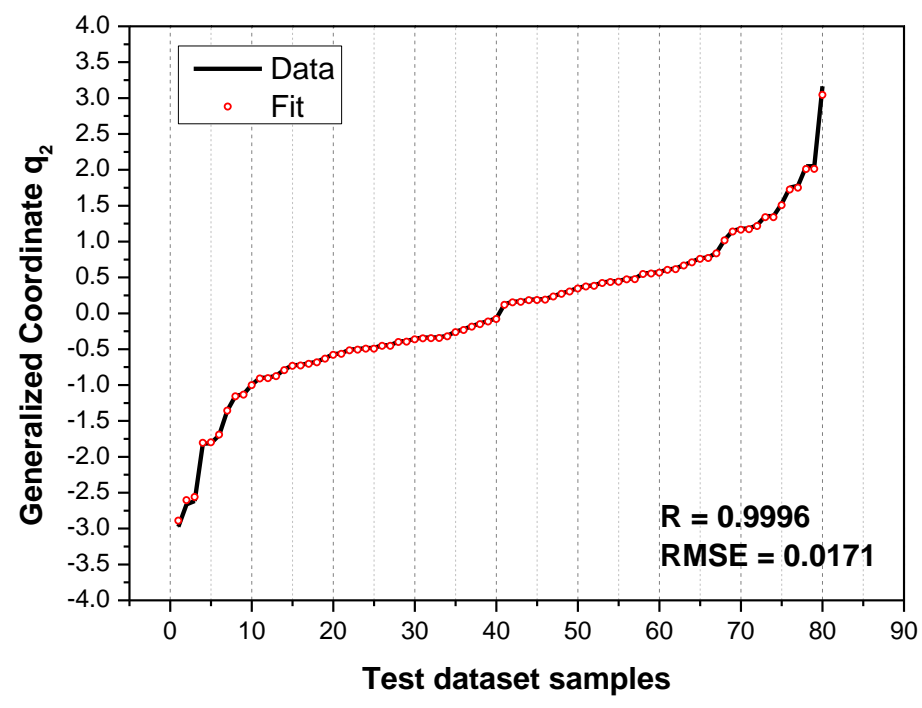

Figure 14. Prediction results of second generalized coordinate.

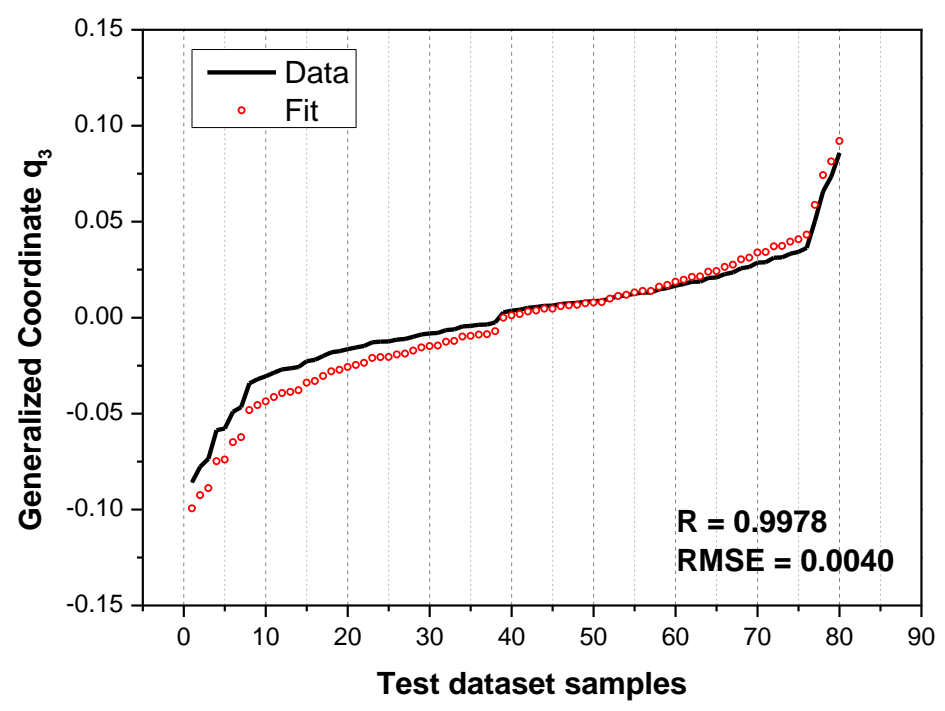

Figure 15. Prediction results of third generalized coordinate. 


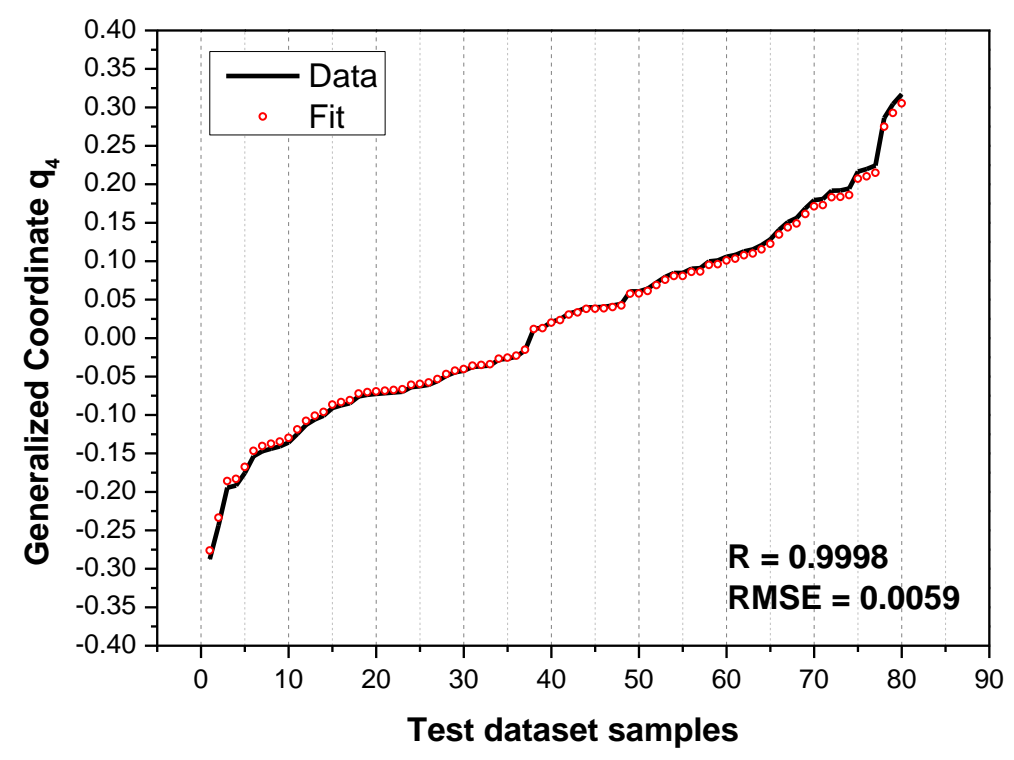

Figure 16. Prediction results of fourth generalized coordinate.

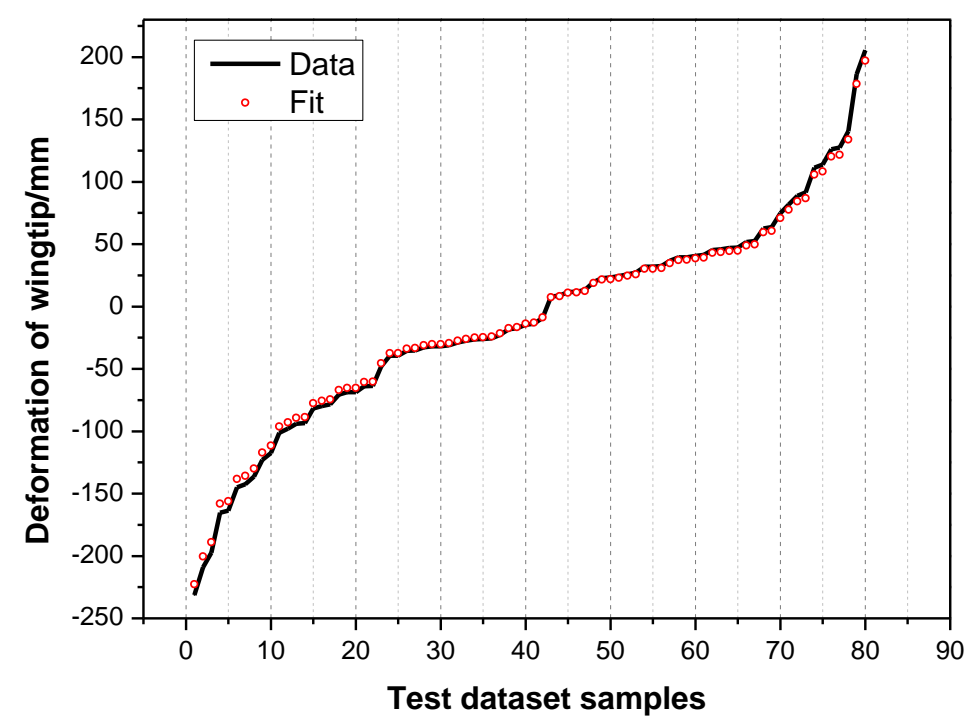

Figure 17. Prediction results of wingtip deformation.

The RFR algorithm is another useful machine learning algorithm for predicting model construction. A random forest (RF) operates by constructing multiple decision trees at different training times and outputting the mean prediction (regression) of individual trees [34]. For comparison, the SVR algorithm in Figure 7 is replaced by the RFR algorithm and the training samples and test dataset samples are in tune with the SVR model. The number of regression trees, $N_{T}$, was set as 50 in model building. Table 3 presents the prediction results of generalized coordinates between the two algorithms. It can be seen that the SVR model had better performance under statistical significance. The prediction model constructed by the SVR algorithm is more accurate for the large deformation calculation problem.

Table 3. Prediction results of the SVR model and the RFR model.

\begin{tabular}{ccccccccc}
\hline \multirow{2}{*}{ Algorithm } & \multicolumn{2}{c}{ First Coordinate } & \multicolumn{2}{c}{ Second Coordinate } & \multicolumn{2}{c}{ Third Coordinate } & \multicolumn{2}{c}{ Fourth Coordinate } \\
\cline { 2 - 9 } & $\mathbf{R}$ & RMSE & $\mathbf{R}$ & RMSE & $\mathbf{R}$ & RMSE & R & RMSE \\
\hline SVR & 0.99971 & 1.8452 & 0.9996 & 0.0171 & 0.9978 & 0.0040 & 0.9998 & 0.0059 \\
RFR & 0.9979 & 2.4135 & 0.9994 & 0.0373 & 0.9974 & 0.0059 & 0.9953 & 0.0153 \\
\hline
\end{tabular}


Although the training and test datasets were generated via FEM calculation, it is notable that the proposed methods are applicable to the real-world datasets. That is also an important practical application of efficient large deformation calculation with a real wing model. The ground test of the real wing model [37] was completed to validate the accuracy of the SVR model proposed.

In the first test, the test model is deformed under self-weight. The wingtip vertical deformation is up to $0.253 \mathrm{~m}$, which is $25.3 \%$ of the span length. The deformation results of measurement and SVR model prediction are listed in Table 4 . The position, $\mathrm{s}$ denotes the location coordinate of measurement on the wing model. Two results reached excellent agreement with deviation up to $1.2 \%$ in the wingtip. Figure 18 shows the deformed beam configuration of this test. The black and red solid lines represent the measurement results and predicted results, respectively.

Table 4. Deformation results of measurement and SVR model in the first test.

\begin{tabular}{cccc}
\hline Position, $\mathbf{s}$ (s = 0 at Root) & Measurement $/ \mathbf{m}$ [37] & SVR model/m & Deviation $\%$ \\
\hline 0.2 & -0.0161 & -0.0162 & 0.62 \\
0.44 & -0.0692 & -0.0697 & 0.72 \\
0.64 & -0.129 & -0.1302 & 0.93 \\
0.8 & -0.182 & -0.1841 & 1.1 \\
1.0 & -0.249 & -0.2519 & 1.2 \\
\hline
\end{tabular}

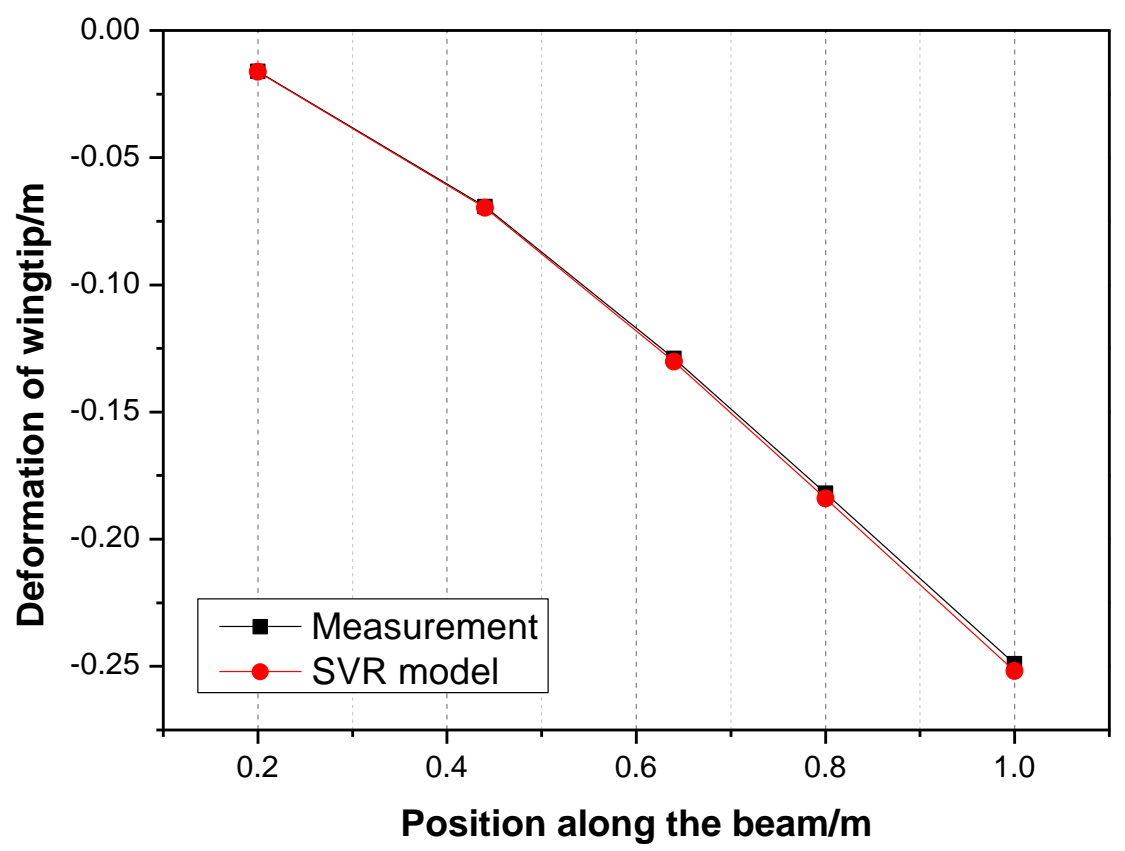

Figure 18. Comparison of measurement and SVR model in the first test.

In the second test, the test model is loaded by a $200 \mathrm{~g}$ weight at the tip. That is equivalent to loading a $1.96 \mathrm{~N}$ tip force on the basis of the first test. The wingtip vertical deformation is up to $0.459 \mathrm{~m}$, which is $45.9 \%$ of the span length. It is much bigger that all sample datasets in the training procedure of the SVR model. The deformation results of measurement and SVR model prediction are listed in Table 5. Figure 19 shows the deformed beam configuration of this test. Deviation up to $4.02 \%$ in the wingtip is larger than the first test. The results are acceptable considering the deformation is far beyond the sample datasets in this test. The generalization capability of the SVR model proposed is excellent and it is very suitable for large deformation prediction of the flexible wing model. 


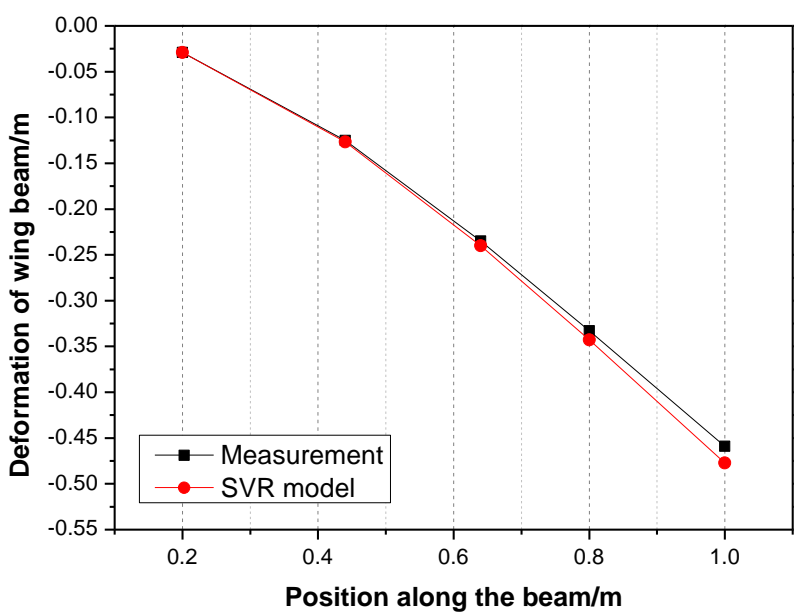

Figure 19. Comparison of measurement and SVR model in the second test.

Table 5. Deformation results of measurement and SVR model in the second test.

\begin{tabular}{cccc}
\hline Position, s (s = 0 at Root) & Measurement $/ \mathbf{m}$ [37] & SVR Model $/ \mathbf{m}$ & Deviation $\%$ \\
\hline 0.2 & -0.0289 & -0.0291 & 0.71 \\
0.44 & -0.125 & -0.1267 & 1.35 \\
0.64 & -0.235 & -0.2400 & 2.11 \\
0.8 & -0.333 & -0.3428 & 2.95 \\
1.0 & -0.459 & -0.4772 & 4.02 \\
\hline
\end{tabular}

Finally, in contrast to the traditional ROM described before, the SVR model presented requires less training samples to achieve the same accuracy. With the same sample datasets presented above, the traditionalt ROM described in Section 2.1 was also established. From 1 to 190 samples were chosen as training samples and the other 270 samples were used as test datasets to evaluate the performance of the prediction model. The RMSE results of vertical deformation of the wingtip are shown in Figure 20. The black solid line describes the SVR model results and the red circle symbol describes the ROM results. It can be seen that RMSE of the SVR model remained at a low level after nearly 30 training samples, but the ROM method required nearly 120 training samples to achieve the same accuracy. Samples in these two structural prediction models' construction are generated in the nonlinear FEM model, which means lots of simulation time will be needed before training models. The SVR model required less training samples with the same accuracy and it will be more efficient and practical in large deformation prediction of flexible wing-like structures.

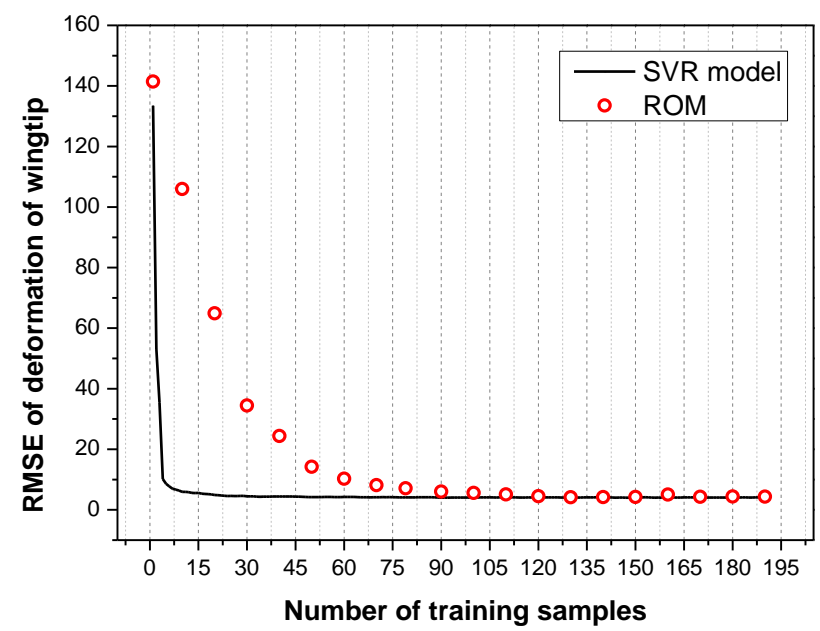

Figure 20. Performance comparison of the SVR model and the traditional reduced-order model (ROM). 


\section{Conclusions}

In this paper, the SVR model was used to predict the static large deformation of flexible wing-like structures. The modal excited load was taken as a feature and the generalized coordinates in modal space were used as the target value. The samples were generated in nonlinear FEM software and divided into training samples $(70 \%)$ and test datasets $(30 \%)$. The correlation coefficient $(\mathrm{R})$ and root mean square error (RMSE) were introduced to evaluate the performance of the SVR models. The prediction results show that the SVR model had excellent performance in large deformation prediction compared to FEM calculation and RFR model results. Meanwhile, it had enough accuracy in real wing model deformation calculation by validating the measurement results in the ground test. Finally, compared to the traditional ROM established with the same samples, the SVR model required less training samples to achieve the same accuracy. The method proposed here is efficient and accurate in large deformation prediction, including geometric nonlinearities.

Author Contributions: Conceptualization, C.A. and C.X.; methodology, C.A.; software, C.A.; validation, C.X., Y.M. and X.S.; resources (ground test), Y.M.; writing-original draft preparation, C.A.; writing-review and editing, C.A. and C.X.; visualization, C.A. and X.S.; project administration, C.Y. All authors have read and agreed to the published version of the manuscript.

Funding: This research received no external funding.

Conflicts of Interest: The authors declare no conflict of interest.

\section{References}

1. Tang, D.M.; Dowell, E.H. Experimental and theoretical study on aeroelastic response of high-aspect-ratio wings. AIAA J. 2001, 39, 1430-1441. [CrossRef]

2. Tsushima, N.; Yokozeki, T.; Su, W.H.; Arizono, H. Geometrically nonlinear static aeroelastic analysis of composite morphing wing with corrugated structures. Aerosp. Sci. Technol. 2019, 88, 244-257. [CrossRef]

3. Sun, M.; Ji, X.Z.; Sun, K.W.; Zhu, M. Flight strategy optimization for high-altitude solar-powered aircraft based on gravity energy reserving and mission altitude. Appl. Sci. 2020, 10, 2243. [CrossRef]

4. Frulla, G.; Cestino, E.; Marzocca, P. Critical behaviour of slender wing configurations. Proc. Imeche Part. G J. Aerosp. Eng. 2009, 224, 587-600. [CrossRef]

5. Liu, Y.; Xie, C.C.; Yang, C.; Cheng, J.L. Gust response analysis and wind tunnel test for a high-aspect ratio wing. Chin. J. Aeronaut. 2016, 29, 91-103. [CrossRef]

6. Nguyen, A.T.; Han, J.H. Wing flexibility effects on the flight performance of an insect-like flapping-wing micro-air vehicle. Aerosp. Sci. Technol. 2018, 79, 468-481. [CrossRef]

7. Liu, Y.Z.; Wan, Z.Q.; Yang, C.; Wang, X.Z. NURBS-Enhanced meshfree method with an integration subtraction technique for complex topology. Appl. Sci. 2020, 10, 2587. [CrossRef]

8. Rezaiee-Pajand, M.; Hashemian, M.; Bohluly, A. A novel time integration formulation for nonlinear dynamic analysis. Appl. Sci. 2020, 10, 2587. [CrossRef]

9. Mignolet, M.P.; Soize, C. Stochastic reduced order models for uncertain geometrically nonlinear dynamical systems. Comput. Methods Appl. Mech. Eng. 2008, 197, 3951-3963. [CrossRef]

10. McEwan, E.I.; Wright, J.R.; Cooper, J.E.; Leung, A.Y.T. A combined modal/finite element analysis technique for the dynamic response of a nonlinear beam to harmonic excitation. J. Sound Vib. 2001, 243, 601-624. [CrossRef]

11. Hollkamp, J.J.; Gordon, R.W.; Cooper, J.E.; Leung, A.Y.T. Reduced-order models for nonlinear response prediction: Implicit condensation and expansion. J. Sound Vib. 2008, 318, 1139-1153. [CrossRef]

12. Przekop, A.; Stover, M.A.; Rizzi, S.A. Nonlinear reduced-order simulation using stress-free and pre-stressed modal bases. In Proceedings of the 50th AIAA/ASME/ASCE/AHS/ASC Structures, Structural Dynamics, and Materials Conference, Palm Springs, CA, USA, 4-7 May 2009.

13. Hollkamp, J.J.; Gordon, R.W.; Spottswood, S.M. Nonlinear modal models for sonic fatigue response prediction: A comparison of methods. J. Sound Vib. 2005, 284, 1145-1163. [CrossRef]

14. An, C.; Yang, C.; Xie, C.C.; Yang, L. Flutter and gust response analysis of a wing model including geometric nonlinearities based on a modified structural ROM. Chin. J. Aeronaut. 2020, 33, 48-63. [CrossRef] 
15. Xie, C.C.; An, C.; Liu, Y.; Yang, C. Static aeroelastic analysis including geometric nonlinearities based on reduced order model. Chin. J. Aeronaut. 2017, 30, 638-650. [CrossRef]

16. Yang, C.; Liang, K.; Rong, Y.F.; Sun, B. A hybrid reduced-order modeling technique for nonlinear structural dynamic simulation. Aerosp. Sci. Technol. 2019, 84, 724-733. [CrossRef]

17. Cestino, E.; Frulla, G.; Marzocca, P. A reduced order model for the aeroelastic analysis of flexible wings. SAE Int. J. Aerosp. 2013, 6, 447-458. [CrossRef]

18. Mignolet, M.P.; Przekop, A.; Rizzi, S.A.; Spottswood, S.M. A review of indirect/nonintrusive reduced order modeling of nonlinear geometric structures. J. Sound Vib. 2013, 332, 62437-62460. [CrossRef]

19. Mei, C. Three decades' interesting experience in nonlinear finite element formulation development and aerospace applications. In Proceedings of the Eighth International Conference on Recent Advances in Structural Dynamics, Southampton, UK, 14-16 July 2003.

20. Spottswood, S.M.; Allemang, R.J. On the investigation of some parameter identification and experimental modal filtering issues for nonlinear reduced order models. Exp. Mech. 2007, 47, 511-521. [CrossRef]

21. Gordon, R.W.; Hollkamp, J.J. Reduced-order Models for Acoustic Response Prediction; Air Force Research Laboratory Report; Air Force Research Laboratory: Wright-Patterson Air Force Base, OH, USA, 2011.

22. Vapnik, V.N. Statistical Learning Theory; Wiley: New York, NY, USA, 1998.

23. Cortes, C.; Vapnik, V.N. Support vector networks. Mach. Learn. 1995, 20, 273-297. [CrossRef]

24. Ozer, S.; Chen, C.; Cirpan, H.A. A set of new Chebyshev kernel functions for support vector machine pattern classification. Pattern Recognit. 2011, 44, 1435-1447. [CrossRef]

25. Tao, D.W.; Ma, Q.; Li, S.L.; Xie, Z.N.; Lin, D.X.; Li, S.Y. Support vector regression for the relationships between ground motion parameters and macroseismic intensity in the sichuan-yunnan region. Appl. Sci. 2020, 10, 3086. [CrossRef]

26. Al-Anazi, A.F.; Gates, I.D. Support vector regression to predict porosity and permeability: Effect of sample size. Comput. Geosci. 2012, 39, 64-76. [CrossRef]

27. Chang, C.C.; Lin, C.J. LIBSVM: A library for support vector machines. ACM Trans. Intell. Syst. Technol. 2011, 2, 27.

28. Liu, H.X.; Li, Q.; Yu, D.B.; Gu, Y. Air quality index and air pollutant concentration prediction based on machine learning algorithms. Appl. Sci. 2019, 9, 4069. [CrossRef]

29. Moradzadeh, A.; Mansour-Saatloo, A.; Mohammadi-Ivatloo, B.; Anvari-Moghaddam, A. Performance evaluation of two machine learningtechniques in heating and cooling loads forecasting of residential buildings. Appl. Sci. 2020, 10, 3829. [CrossRef]

30. Cheng, K.; Lu, Z.Z. Adaptive sparse polynomial chaos expansions for global sensitivity analysis based on support vector regression. Comput. Struct. 2018, 194, 86-96. [CrossRef]

31. Clarke, S.M.; Griebsch, J.H.; Simpson, T.W. Analysis of support vector regression for approximation of complex engineering analyses. J. Mech. Des. 2005, 127, 1077-1087. [CrossRef]

32. Santamaria-Bonfil, G.; Reyes-Ballesteros, A.; Gershenson, C. Wind speed forecasting for wind farms: A method based on support vector regression. Renew. Energy 2015, 85, 790-809. [CrossRef]

33. Li, L.; Zheng, W.; Wang, Y. Prediction of moment redistribution in statically indeterminate reinforced concrete structures using artificial neural network and support vector regression. Appl. Sci. 2019, 9, 28. [CrossRef]

34. Leo, B. Random forests. Mach. Learn. 2001, 45, 5-32.

35. Bishop, C. Pattern Recognition and Machine Learning; Springer: New York, NY, USA, 2006.

36. Zhang, J.; Liao, Y.; Wang, S.; Han, J. Study on driving decision-making mechanism of autonomous vehicle. Appl. Sci. 2018, 8, 13. [CrossRef]

37. Meng, Y.; Xie, C.C.; Wan, Z.Q. Strain-based shape prediction for flexible beam-like structures. In Proceedings of the AIAA Scitech 2019 Forum, San Diego, CA, USA, 7-11 January 2019; AIAA: Reston, VA, USA, 2019.

38. Meng, Y.; Xie, C.C.; Wan, Z.Q. Deformed Wing Shape Prediction Using Fiber Optic Strain Data. In Proceedings of the 17th International Forum on Aeroelasticity and Structural Dynamics, Como, Italy, 25-28 June 2017.

(C) 2020 by the authors. Licensee MDPI, Basel, Switzerland. This article is an open access article distributed under the terms and conditions of the Creative Commons Attribution (CC BY) license (http://creativecommons.org/licenses/by/4.0/). 